\title{
Choline Deficiency Attenuates Body Weight Gain and Improves Glucose Tolerance in ob/ob Mice
}

\author{
Gengshu Wu, ${ }^{1}$ Liyan Zhang, ${ }^{2}$ Tete Li, ${ }^{1}$ Gary Lopaschuk, ${ }^{2}$ \\ Dennis E. Vance, ${ }^{1}$ and René L. Jacobs ${ }^{3}$ \\ ${ }^{1}$ Group on the Molecular and Cell Biology of Lipids and Department of Biochemistry, University of Alberta, \\ Edmonton, AB, Canada T6G $2 S 2$ \\ ${ }^{2}$ Cardiovascular Research Group, University of Alberta, Edmonton, AB, Canada T6G 2S2 \\ ${ }^{3}$ Department of Agricultural, Food and Nutritional Sciences, University of Alberta, Edmonton, AB, Canada T6G $2 S 2$ \\ Correspondence should be addressed to Dennis E. Vance, dennis.vance@ualberta.ca
}

Received 1 November 2011; Revised 2 February 2012; Accepted 16 February 2012

Academic Editor: Bernhard Breier

Copyright (C) 2012 Gengshu Wu et al. This is an open access article distributed under the Creative Commons Attribution License, which permits unrestricted use, distribution, and reproduction in any medium, provided the original work is properly cited.

Previous studies demonstrated that choline supply is directly linked to high-fat-diet-induced obesity and insulin resistance in mice. The aim of this study was to evaluate if choline supply could also modulate obesity and insulin resistance caused by a genetic defect. Eight-week-old male ob/ob mice were fed for two months with either choline-deficient or choline-supplemented diet. Tissue weight including fat mass and lean mass was assessed. Intracellular signaling, plasma glucagon and insulin, and glucose and insulin tolerance tests were also investigated. The choline-deficient diet slowed body weight gain and decreased fat mass. Choline deficiency also decreased plasma glucose level and improved glucose and insulin tolerance although fatty liver was exacerbated. Increased adipose lipolytic activity, decreased plasma glucagon and reduced expression of hepatic glucagon receptor were also observed with the choline-deficient diet. Our results demonstrate that a choline-deficient diet can decrease fat mass and improve glucose tolerance in obese and diabetic mice caused by a genetic defect.

\section{Introduction}

Choline is an important dietary factor, which is involved in some crucial processes such as the biosynthesis of neurotransmitter acetylcholine and the major membrane component phosphatidylcholine (PC) $[1,2]$. PC can be synthesized either by the CDP-choline pathway or via methylation of phosphatidylethanolamine by phosphatidylethanolamine $\mathrm{N}$ methyltransferase (PEMT). The PEMT pathway is only quantitatively significant in liver $[3,4]$. Choline is also an essential precursor of the neurotransmitter acetylcholine, which acts through muscarinic and nicotinic acetylcholine receptors. In recent years, a possible link between choline and obesity/diabetes has become apparent $[5,6]$. The potential of muscarinic acetylcholine receptors as a therapeutic target of obesity has been proposed [7-9]. Recently, we found that PEMT deficiency could protect mice from high-fatdiet-induced obesity and insulin resistance. However, this protection disappeared when a high dose of choline was added to the high-fat-diet [5]. The PEMT pathway followed by PC catabolism is the only known biosynthetic pathway for choline in mammals [10]. A significant decrease of choline content in several tissues was found in PEMT-deficient mice ( $\mathrm{Li}$ et al., unpublished data) providing a direct link between choline and high-fat-diet-induced obesity/insulin resistance. In the current study, we fed ob/ob mice a choline deficient (CD) diet to evaluate if choline deficiency could also modulate genetic-derived obesity and insulin resistance.

\section{Material and Methods}

2.1. Animals and Diet. All procedures were approved by University of Alberta's Institutional Animal Care Committee in accordance with guidelines of the Canadian Council on Animal Care. 8-week-old male ob/ob mice from The Jackson Laboratory were acclimated for 1 week. 5 mice in each group were used. The mice had free access to choline deficient (CD) or choline-supplemented (CS) diets for 2 months. 
The CD diet (MP Biomedicals Canada, catalog no. 901387) contained $20 \%$ fat as lard. The CS diet consisted of the CD diet to which was added $0.4 \%(\mathrm{w} / \mathrm{w})$ choline chloride. Mice were sacrificed by anaesthetizing with isoflurane, followed by heart puncture.

\subsection{Body Weight Gain, Lean Mass and Fat Mass Measurement.} The body weight was measured weekly. The lean mass and fat mass were analyzed, 2 months after mice were put on diet (premortem), using the EchoMRI system.

2.3. Glucose and Insulin Tolerance Test. Glucose tolerance test: following a 12-hour fast, mice received an intraperitoneal injection (i.p.) of $0.5 \mathrm{~g}$ of dextrose $/ \mathrm{kg}$ body weight dissolved in sterilized phosphate buffered saline (PBS). Insulin tolerance test: following a 6-hour fast, mice received an i.p. injection of 1.0 unit of insulin (Sigma) $/ \mathrm{kg}$ body weight in PBS. Blood glucose concentrations were measured by a glucometer in tail bleeds before and at times indicated after injection.

\subsection{Measurements of Mitochondrial Complex I/II Activity.} The activities of mitochondrial complex I and II were determined by using mitochondrial protein. Briefly, to isolate mitochondrial protein, samples were kept on ice and homogenized in a buffer containing $75 \mathrm{mM}$ sucrose, $225 \mathrm{mM}$ sorbitol, $1 \mathrm{mM}$ EGTA, and $0.1 \%$ fatty acid-free bovine serum albumin in $10 \mathrm{mM}$ Tris- $\mathrm{HCl} \mathrm{pH}$ 7.4. The homogenate was centrifuged at $600 \times \mathrm{g}$ for $15 \mathrm{~min}$, and the resulting supernatant was further centrifuged for $20 \mathrm{~min}$ at $16,200 \mathrm{rpm}$. The mitochondrial protein in the pellets was suspended in a buffer containing $20 \mathrm{mM}$ Tris and $25 \mathrm{mM}$ Sucrose (pH7.4) and kept at $-80^{\circ} \mathrm{C}$ until used. The measurement of activity was performed in a spectrophotometer for a period of 5 min as a decrease in the absorbance at $600 \mathrm{~nm}$ due to the reduction of 2,6-dichlorophenol-indophenol (DCPIP) to ubiquinone- 1 in the presence of either NADH (substrate of complex I) or succinate (substrate of complex II) accordingly. The reaction was carried out in the presence of antimycin and potassium cyanide as inhibitor of complex III and complex IV. Rotenone, as inhibitor of complex I, was also involved in the reaction buffer for complex II activity.

2.5. Histology and Immunoblotting. For histology, the livers or fat pads were dissected into $10 \%$ formaldehyde in PBS, then followed by the routine hematoxylin and eosin staining. For immunoblotting, following the homogenization and centrifugation at $600 \times \mathrm{g}$ of the liver samples, the resulting supernatants were subjected to protein concentration determination (BioRad) for ensuring equal amount of proteins was loaded to SDS-PAGE. All primary antibodies against hormone-sensitive lipase (phospho-HSL/total HSL), phosphoenolpyruvate carboxykinase, (PEPCK), Acetyl-CoA Carboxylase (phospho-ACC/total ACC), and AMP-activated protein kinase (phospho-AMPK/total AMPK) are purchased from Cell Signaling. Antibodies to pyruvate dehydrogenase kinase isozyme 4 (PDK4), glucagon receptor were obtained from Abcam, but suppressor of cytokine signaling 3 (SOCS3) and PGC- $1 \alpha$ were from Santa Cruz Biotechnology.Tublin or actin was used as loading controls for normalization. Image was analyzed by ImageJ software.

2.6. Plasma Insulin and Glucagon Measurement. The measurements were performed using a mouse/rat glucagon, insulin assay kit (Meso Scale Discovery) based on the company's instructions.

2.7. Statistical Analysis. Data are shown as mean \pm SD. The differences were assessed using the student's $t$-test. A value of $P<0.05$ was considered significant.

\section{Results}

Choline-deficient diet significantly slowed body weight gain up to 2 months (Figure 1(a)) without influencing food consumption (daily food intake $7.21 \pm 1.23 \mathrm{~g}$ for CD mice versus $7.39 \pm 1.31 \mathrm{~g}$ for CS mice, $P>0.05)$. There was significant difference in starting body weight between two groups $(30.42 \pm 1.70 \mathrm{~g}$ for CD mice versus $26.3 \pm 2.26 \mathrm{~g}$ for CS mice, $P=0.01$ ). Choline deficiency didnot influence the weight of heart, liver, or kidney (Figure 1(b)), but there was a significant decrease in the weight of epididymal and perirenal fat (Figure 1(c)). Further analysis, using magnetic resonance imaging, showed that choline deficiency decreased whole body fat mass and yet increased lean mass no matter expressed as absolute mass or relative percentage to whole body mass (Figure 1(d)). Choline deficiency increased expression of adipose tissue phosphohormone-sensitive lipase, an activated form of the enzyme (Figure 1(e)). Choline deficiency, on the other hand, did not change the morphology of adipose tissue (Figure 1(f)).

Besides influencing body weight, choline deficiency also decreased fasting plasma glucose $(14.3 \pm 1.67$ versus $10.8 \pm 1.19, n=5, P<0.05)$ and glucagon level (Figure 2(a)), although it had no effect on plasma insulin level (Figure 2(b)). A significantly improved glucose and insulin intolerance were observed in mice on cholinedeficient diet (Figures 2(c)-2(d)). To investigate the underlying mechanism, the key enzymes for glucose oxidation were analysed. Choline deficiency reduced hepatic protein expression of PDK4 (Figure 3(a)), a negative regulator of pyruvate dehydrogenase activity that controls the influx of mitochondrial acetyl-CoA, which indicates an enhanced glucose utilization. Similarly, hepatic PGC-1 $\alpha$ (a well-known upstream modulator of PDK4) and PEPCK (a downstream target of PGC-1 $\alpha$ and a key enzyme for gluconeogenesis) were reduced (Figure $3(\mathrm{~b})$ ), suggesting a diminished gluconeogenesis. Moreover, we investigated if the improved glucose and insulin intolerance could be associated with the alteration of hepatic inflammation and mitochondrial function. Neither the protein expression of SOCS3 (Figure 3(c)), a marker of proinflammation, nor the activity of mitochondrial citrate synthase was affected (data not shown). However, the activity of mitochondrial complex II, but not complex I, was significantly decreased by choline deficiency (Figure 3(d)). Of interest, protein expression of the hepatic 


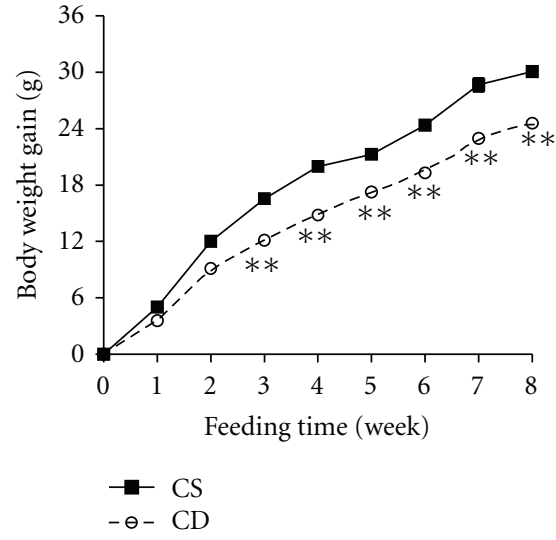

(a)
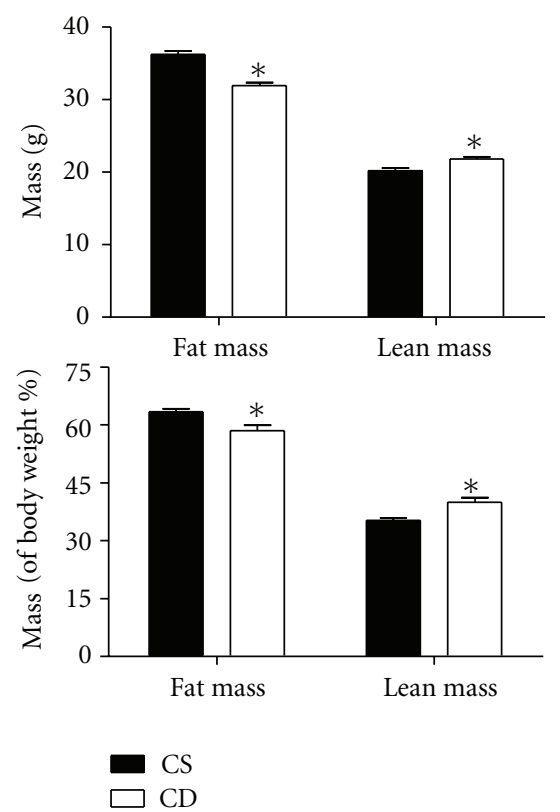

(d)

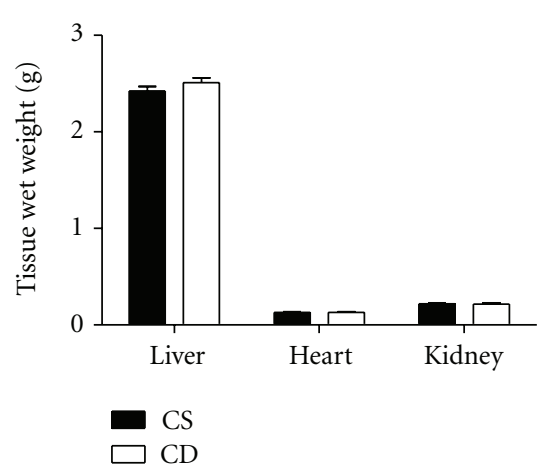

(b)

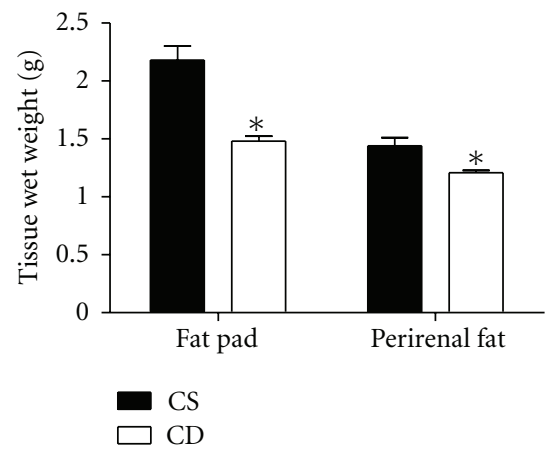

(c)

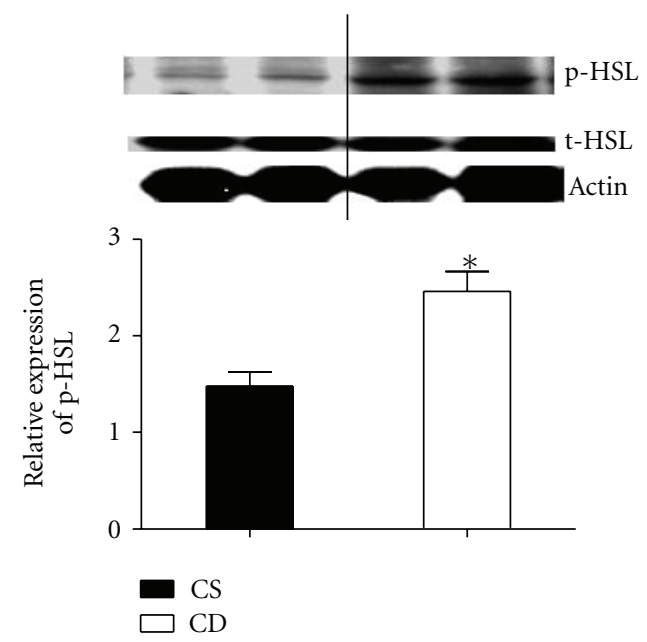

(e)
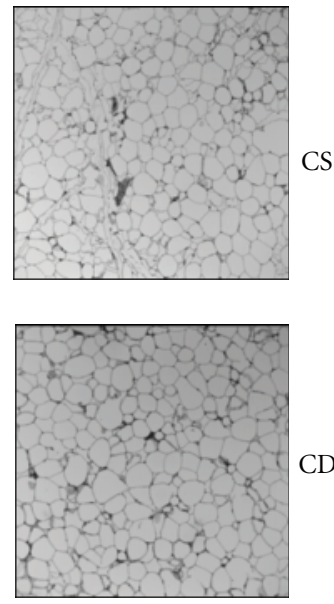

(f)

Figure 1: Choline-deficient diet attenuates body weight gain and enhances adipose lipolytic capacity. 8-week-old male ob/ob mice had free access to either CD or CS diets for 2 months. Body weight gain (a), tissue weight (b), epididymal and perirenal fat weight (c), and fat mass and lean mass (d) were measured. The protein expression of phospho- and total-hormone sensitive lipase in adipose tissue was assayed by immunoblotting (e). The fat pad sections were stained with $\mathrm{H} \& \mathrm{E}(\mathrm{f}) .{ }^{* *} P<0.01$ and ${ }^{*} P<0.05$ when compared to CS group.

glucagon receptor was also decreased by choline deficiency (Figure 3(e)).

Although improving glucose tolerance, choline deficiency exacerbates fatty liver, supported by histological analysis and increased hepatic triglyceride mass (Figure 4(a)). To understand the mechanisms underlying choline deficiencyinduced fatty liver, we looked into the enzymes involved in fatty acid $\beta$-oxidation and lipogenesis. Choline deficiency increased hepatic glycerol-3-phospho-acyltransferase (GPAT) activity (Figure 4(b)) and decreased the activity of $\beta$-hydroxyacyl-CoA dehydrogenase ( $\beta$-HAD) (Figure 4(c)), a key enzyme of fatty acid $\beta$-oxidation, which indicates that more intracellular fatty acids were channeled into lipid biosynthesis rather than oxidation. This notion was further supported by a decrease in hepatic phospho-AMPK and
phospho-ACC expression (Figure 4(d)), which then activates ACC and enhances hepatic lipogenesis.

\section{Discussion}

In previous work, we have demonstrated that choline supplementation is linked to high-fat-diet-induced obesity and insulin resistance in PEMT-deficient mice [5]. Recently, we have found that choline-induced insulin resistance is choline specific, not only in wild-type mice but also in PEMT deficient mice (Wu and Vance et al. unpublished data). In the current study, we investigated the impact of choline deprivation on ob/ob mice. We demonstrated five novel effects of choline deficiency: (1) increased adipose lipolytic activity; (2) improved glucose oxidation; (3) decreased hepatic fatty 


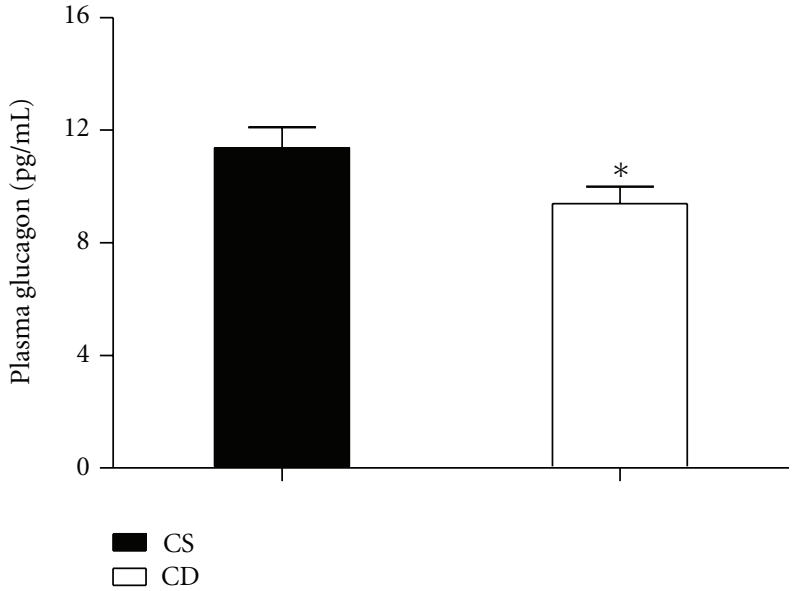

(a)

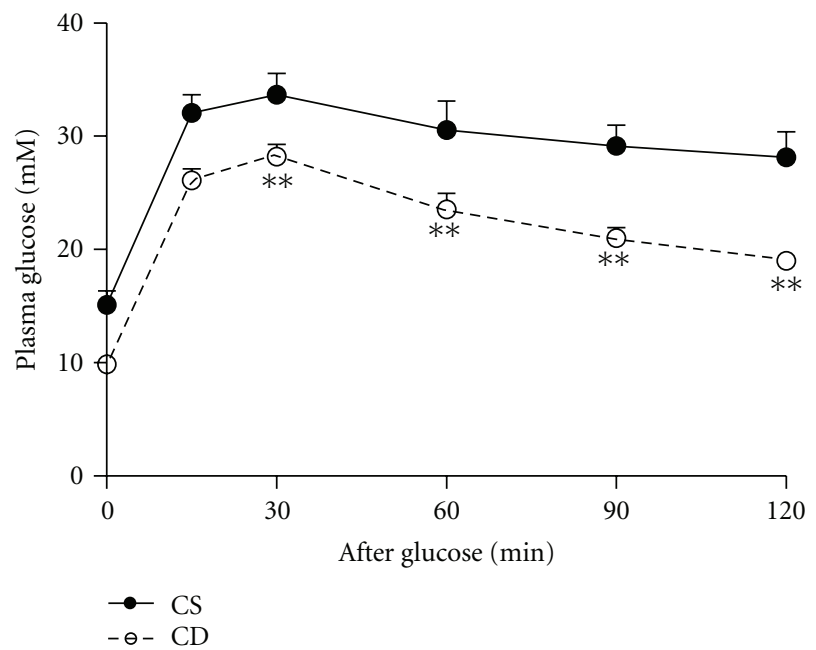

(c)

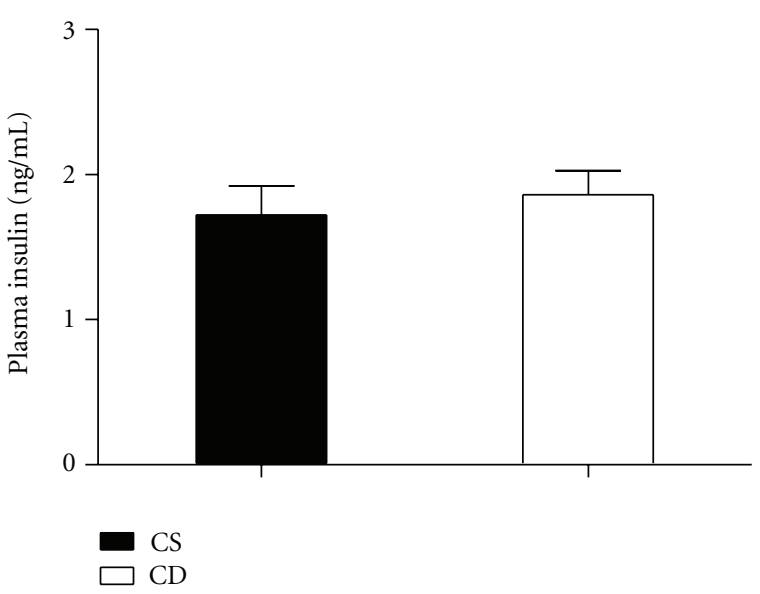

(b)

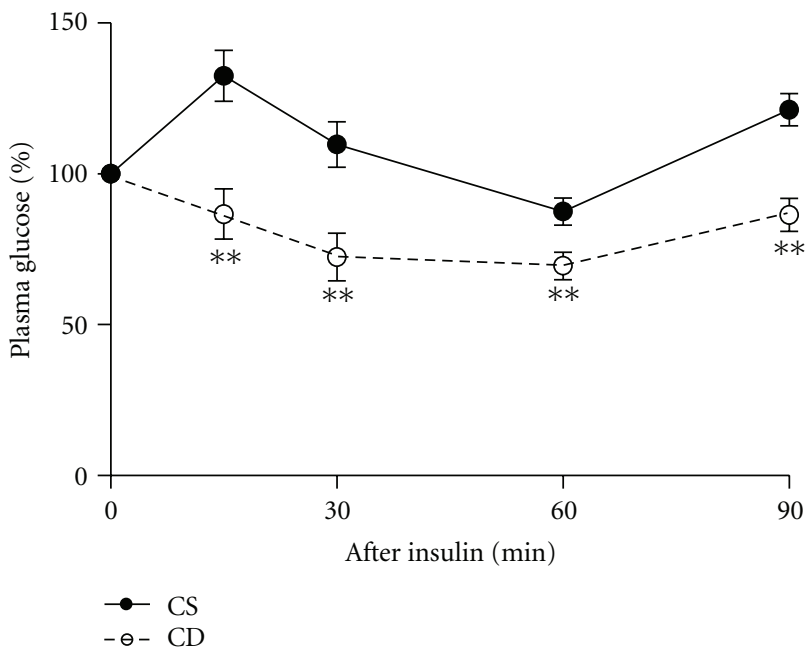

(d)

FIGURE 2: Choline-deficient diet improves glucose and insulin intolerance. 8-week-old male ob/ob mice had free access to either CD or CS diets for 2 months. The level of fasting plasma glucagon (a) and insulin (b) was measured. Intraperitoneal glucose tolerance test (c) and insulin tolerance test $(\mathrm{d})$ were performed. ${ }^{* *} P<0.01$ and ${ }^{*} P<0.05$ when compared to CS group.

acid oxidation; (4) downregulated hepatic glucagon receptor expression; (5) reduced hepatic mitochondrial complex II activity.

4.1. Choline Deficiency Attenuates Body Weight Gain. Choline deficiency significantly attenuates high-fat-diet-induced obesity in $\mathrm{Pemt}^{-/-}$mice and weight gain in ob/ob mice. However, Raubenheimer et al. reported that choline deficiency did not affect weight gain or the weight of adipose tissue [11]. The difference between two studies could be due to differences in the animal models and the feeding protocols. In Raubenheimer's study, C57Bl/6 mice were fed either highfat or low-fat diet for 8 weeks, but mice only received the $\mathrm{CD}$ or CS diet for the last 4 weeks. Our ob/ob mice were fed the high-fat CD or CS diet for 8 weeks. In the literature, both male and female ob/ob mice have been used extensively in metabolic studies, such as body weight gain and hepatic (and muscle) insulin sensitivity, and no significant gender effect has been reported. In our study, only male ob/ob mice were used.

Our current study demonstrates that choline can also modulate genetic-derived obesity. Since choline deficiency did not influence food consumption, increased adipose lipolytic activity (enhanced expression of active hormonesensitive lipase) may explain reduced fat mass and body weight gain. In addition, we cannot exclude the possibilities that choline deficiency could enhance energy expenditure or the expression of UCP1 in brown adipose tissue and UCP2/UCP3 in skeletal muscle, which are potential factors contributing to decreased body weight gain [12].

4.2. Choline Deficiency Improves Glucose Tolerance. In contrast to choline supplementation, which is linked to highfat-diet-induced insulin resistance in mice [5], choline deficiency improved glucose tolerance in ob/ob mice. This is consistent with Raubenheimer's study, where C57Bl/6 


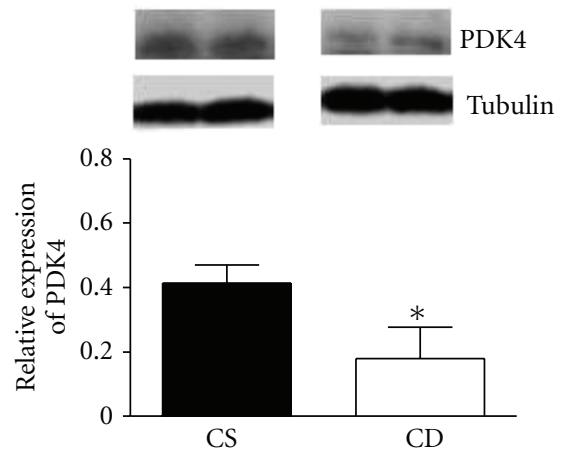

(a)

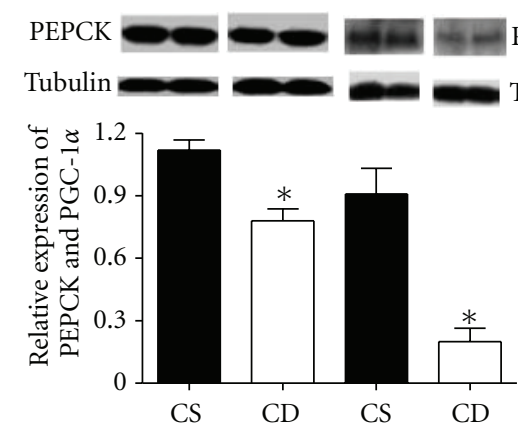

(b)

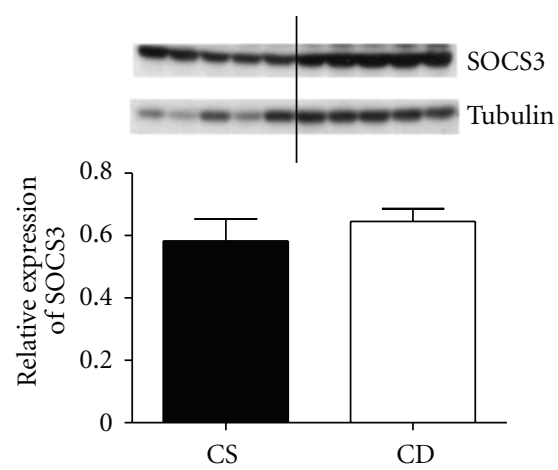

(c)

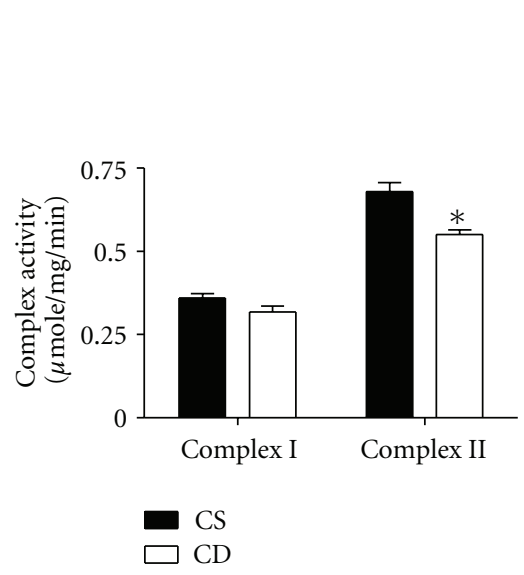

(d)

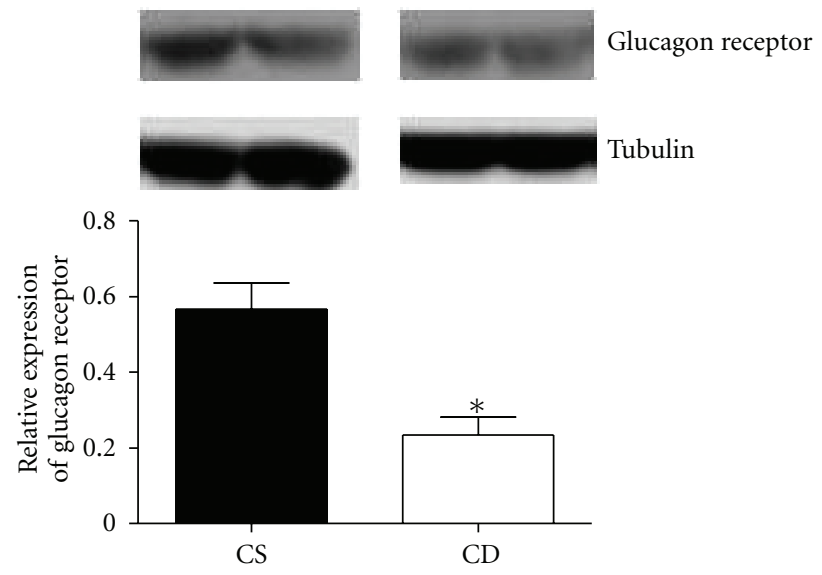

(e)

Figure 3: Molecular mechanisms involved in improved insulin sensitivity in ob/ob mice on choline-deficient diet. 8-week-old male ob/ob mice had free access to either CD or CS diets for 2 months. Hepatic protein expression of PDK4 (a), PEPCK, PGC-1 $\alpha$ (b), SOCS3 (c), and glucagon receptor (e) was assayed by immunoblotting. The activities of mitochondrial complex I and II were also measured (d). ${ }^{*} P<0.05$ when compared to CS group.

mice on a CD high-fat-diet had decreased insulin levels and improved glucose tolerance compared to choline supplemented high-fat diet [11]. High plasma glucagon is usually found in type 2 diabetic patients [13]. Choline injection increased both plasma insulin and glucagon levels in rats [14, 15]. Conversely, choline-deficiency in our study decreased plasma glucagon and decreased expression of the glucagon receptor. Since in recent years glucagon receptor antagonists have been proposed as the potential therapeutic drugs for type 2 diabetes [16, 17], so decreased protein expression of glucagon receptor in ob/ob mice on choline deficient diet, to some extent, may explain the improved glucose tolerance. The decreased hepatic PGC- $1 \alpha$ expression was concomitant with a downregulation of PEPCK and PDK4, indicating a decreased glucose output and increased glucose oxidation. Down-regulation of $\beta$-HADH activity suggested decreased fatty acid oxidation. These processes were accompanied by a diminished mitochondrial complex II activity, which may reflect a compensatory response to reduced mitochondrial oxidative stress. Thus, CD-improved glucose tolerance was due to decreased hepatic glucose output and increased glucose utilization. This could occur through suppression of hepatic glucagon receptor, which regulates AMPK via adenylate cyclase, thereby modulating PGC- $1 \alpha$ and its downstream targets, such as PEPCK and PDK4, as well as hepatic mitochondrial electron transport capacity. Thus, further investigations are needed to prove whether glucagon receptor could be a new therapeutic target for diabetes.

\subsection{The Disconnection between Fatty Liver and Insulin} Resistance. Fatty liver has been shown to be an obesityindependent predictor of type 2 diabetes. Increased intracellular fatty acids are thought to play a causal role in hepatic insulin resistance [18]. In the current study, choline deficiency exacerbated fatty liver, in which a decrease in $\beta$ hydroxyacyl CoA dehydrogenase activity and an increase in glycerol palmitoyl-acyl transferase activity were observed, suggesting a role of choline in modulating fatty acid metabolism. Hence, redirection of fatty acids into hepatic triglyceride storage may be an initial protective mechanism to lower hepatic intracellular fatty acid concentrations. Long-term choline deficiency, however, is connected to hepatosteatosis, risk of neural tube defect, as well as the risk of cancer, and memory loss $[1,19,20]$, which may limit 


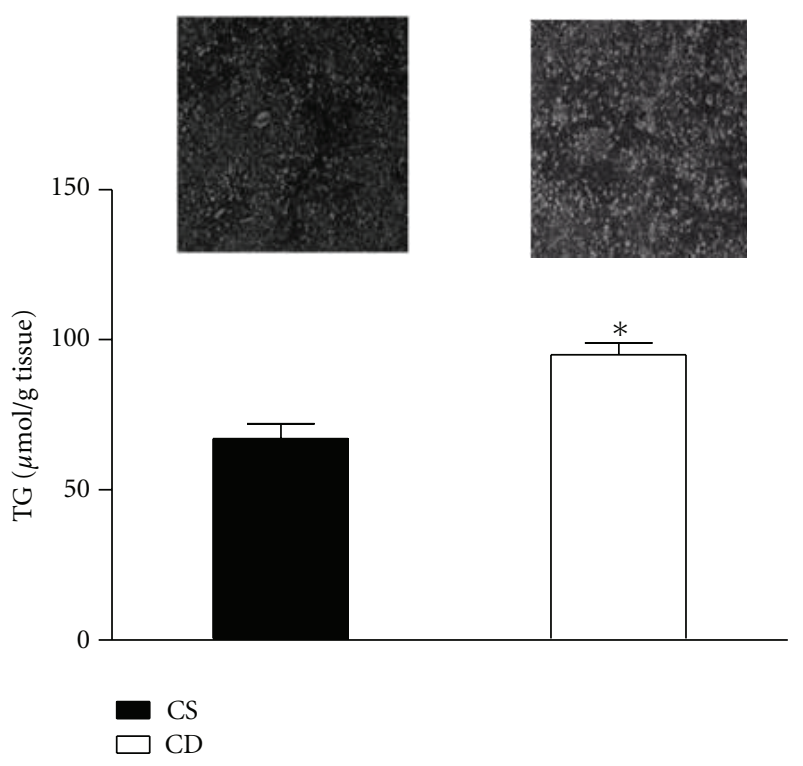

(a)

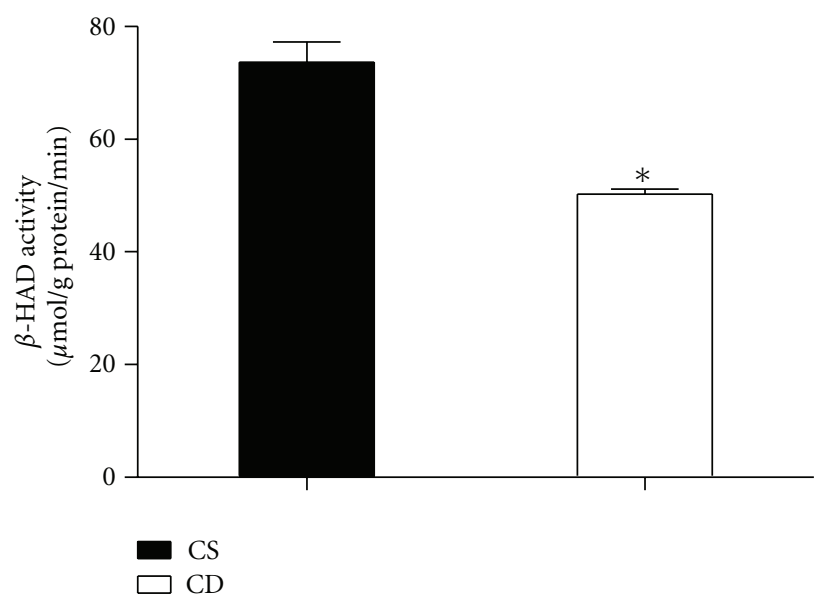

(c)

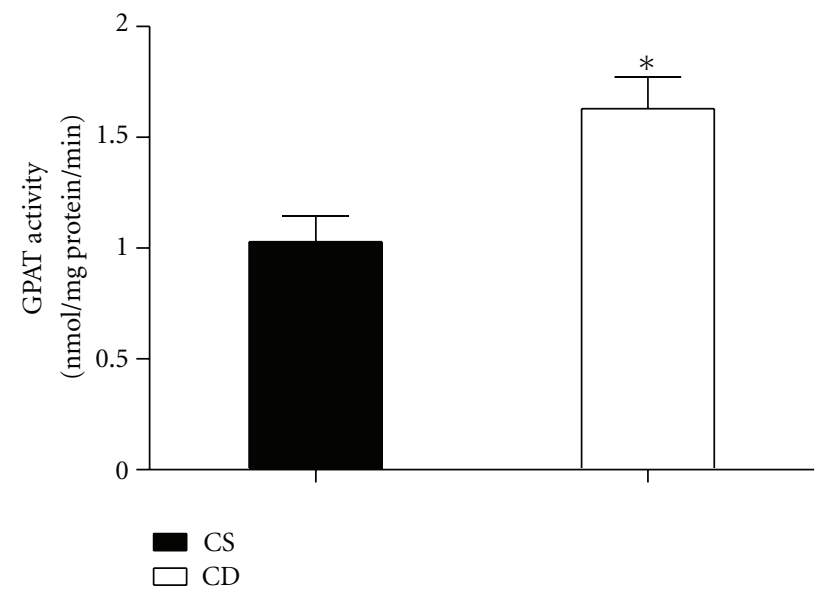

(b)
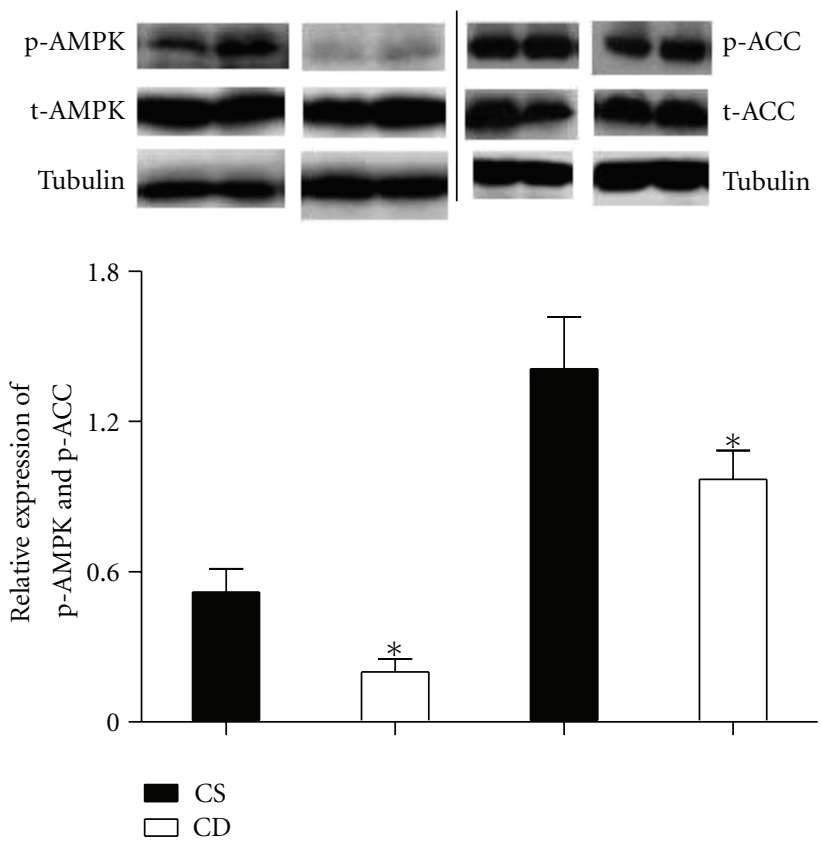

(d)

Figure 4: Molecular mechanisms involved in the development of fatty liver in ob/ob mice on choline-deficient diet. 8-week-old male ob/ob mice had free access to either CD or CS diets for 2 months. The liver sections were stained with $\mathrm{H}$ \& E staining protocol, and the amount of hepatic triglyceride was measured (a). The activities of glycerol-3-phosphate-acyltransferase (GPAT) (b) and $\beta$-hydroxyl acylCoA dehydrogenase $\left(\beta\right.$-HAD) $(\mathrm{c})$ were assayed. The protein expression of phospho-AMPK and phospho-ACC was monitored $(\mathrm{d}) .{ }^{*} P<0.05$ when compared to CS group.

the use of a choline-deficient diet as a long-term therapeutic strategy for obesity and insulin resistance.

In conclusion, choline deficiency can prevent body weight gain and improve glucose tolerance in ob/ob mice. Modest restriction of high-choline-containing food intake may benefit obesity, prediabetic, and diabetic patients. Our results suggest novel possible therapeutic approaches.

\section{Abbreviations}

PC: Phosphatidylcholine

PEMT: Phosphatidylethanolamine

$\mathrm{N}$-methyltransferase

CD: Choline deficient

CS: Choline supplement

HSL: Hormone sensitive lipase 
PEPCK: Phosphoenolpyruvate carboxykinase

ACC: Acetyl-CoA Carboxylase

AMPK: AMP-activated protein kinase (AMPK)

PDK4: Pyruvate dehydrogenase kinase isozyme 4

SOCS3: Suppressor of cytokine signaling 3

GPAT: Glycerol-3-phospho-acyltransferase.

\section{Authors' Contribution}

G. Wu and L. Zhang contributed equally.

\section{Author Disclosure}

The authors declare no conflict of interest.

\section{Acknowledgments}

This work was supported by a grant (MOP 89793) from the Canadian Institutes of Health Research.

\section{References}

[1] S. H. Zeisel, "Nutritional importance of choline for brain development," Journal of the American College of Nutrition, vol. 23, no. 6, pp. 621S-626S, 2004.

[2] C. B. Hollenbeck, "The Importance of Being Choline," Journal of the American Dietetic Association, vol. 110, no. 8, pp. 1162$1165,2010$.

[3] D. E. Vance and N. D. Ridgway, "The methylation of phosphatidylethanolamine," Progress in Lipid Research, vol. 27, no. 1, pp. 61-79, 1988.

[4] C. J. Walkey, L. R. Donohue, R. Bronson, L. B. Agellon, and D. E. Vance, "Disruption of the murine gene encoding phosphatidylethanolamine N-methyltransferase," Proceedings of the National Academy of Sciences of the United States of America, vol. 94, no. 24, pp. 12880-12885, 1997.

[5] R. L. Jacobs, Y. Zhao, D. P. Y. Koonen et al., "Impaired de novo choline synthesis explains why phosphatidylethanolamine $\mathrm{N}$ methyltransferase-deficient mice are protected from dietinduced obesity," Journal of Biological Chemistry, vol. 285, no. 29, pp. 22403-22413, 2010.

[6] I. Rubio-Aliaga, B. de Roos, M. Sailer et al., "Alterations in hepatic one-carbon metabolism and related pathways following a high-fat dietary intervention," Physiological Genomics, vol. 43 , no. 8, pp. 408-416, 2011.

[7] A. Maresca and C. T. Supuran, "Muscarinic acetylcholine receptors as therapeutic targets for obesity," Expert Opinion on Therapeutic Targets, vol. 12, no. 9, pp. 1167-1175, 2008.

[8] M. B. Marrero, R. Lucas, C. Salet et al., "An $\alpha 7$ nicotinic acetylcholine receptor-selective agonist reduces weight gain and metabolic changes in a mouse model of diabetes," Journal of Pharmacology and Experimental Therapeutics, vol. 332, no. 1, pp. 173-180, 2010.

[9] D. Gautam, O. Gavrilova, J. Jeon et al., "Beneficial metabolic effects of M3 muscarinic acetylcholine receptor deficiency," Cell Metabolism, vol. 4, no. 5, pp. 363-375, 2006.

[10] Z. Li and D. E. Vance, "Phosphatidylcholine and choline homeostasis," Journal of Lipid Research, vol. 49, no. 6, pp. 1187-1194, 2008.

[11] P. J. Raubenheimer, M. J. Nyirenda, and B. R. Walker, "A choline-deficient diet exacerbates fatty liver but attenuates insulin resistance and glucose intolerance in mice fed a highfat diet," Diabetes, vol. 55, no. 7, pp. 2015-2020, 2006.

[12] H. M. Feldmann, V. Golozoubova, B. Cannon, and J. Nedergaard, "UCP1 ablation induces obesity and abolishes dietinduced thermogenesis in mice exempt from thermal stress by living at thermoneutrality," Cell Metabolism, vol. 9, no. 2, pp. 203-209, 2009.

[13] E. Henkel, M. Menschikowski, C. Koehler, W. Leonhardt, and M. Hanefeld, "Impact of glucagon response on postprandial hyperglycemia in men with impaired glucose tolerance and type 2 diabetes mellitus," Metabolism, vol. 54, no. 9, pp. 11681173, 2005.

[14] Y. O. Ilcol, M. Cansev, M. S. Yilmaz, E. Hamurtekin, and I. H. Ulus, "Peripheral administration of CDP-choline and its cholinergic metabolites increases serum insulin: muscarinic and nicotinic acetylcholine receptors are both involved in their actions," Neuroscience Letters, vol. 431, no. 1, pp. 71-76, 2008.

[15] M. Cansev, Y. O. Ilcol, M. S. Yilmaz, E. Hamurtekin, and I. H. Ulus, "Choline, CDP-choline or phosphocholine increases plasma glucagon in rats: involvement of the peripheral autonomic nervous system," European Journal of Pharmacology, vol. 589, no. 1-3, pp. 315-322, 2008.

[16] S. W. Djuric, N. Grihalde, and C. W. Lin, "Glucagon receptor antagonists for the treatment of type II diabetes: current prospects," Current Opinion in Investigational Drugs, vol. 3, no. 11, pp. 1617-1623, 2002.

[17] W. Gu, D. J. Lloyd, N. Chinookswong et al., "Pharmacological targeting of glucagon and glucagon-like peptide 1 receptors has different effects on energy state and glucose homeostasis in diet-induced obese mice," Journal of Pharmacology and Experimental Therapeutics, vol. 338, no. 1, pp. 70-81, 2011.

[18] D. Gao, S. Nong, X. Huang et al., "The effects of palmitate on hepatic insulin resistance are mediated by NADPH oxidase 3derived reactive oxygen species through JNK and p38 MAPK pathways," Journal of Biological Chemistry, vol. 285, no. 39, pp. 29965-29973, 2010.

[19] A. K. Ghoshal and E. Farber, "Choline deficiency, lipotrope deficiency and the development of liver disease including liver cancer: a new perspective," Laboratory Investigation, vol. 68, no. 3, pp. 255-260, 1993.

[20] Y. K. J. Zhang, R. L. Yeager, Y. Tanaka, and C. D. Klaassen, "Enhanced expression of Nrf2 in mice attenuates the fatty liver produced by a methionine- and choline-deficient diet," Toxicology and Applied Pharmacology, vol. 245, no. 3, pp. 326334, 2010. 


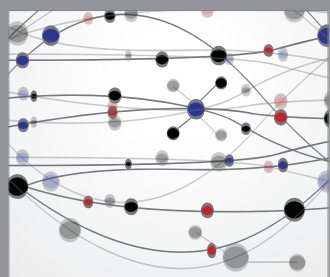

The Scientific World Journal
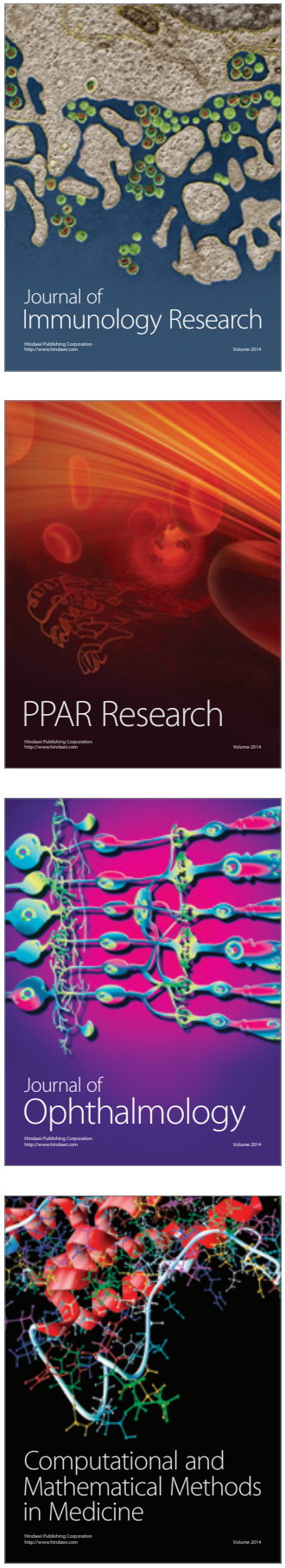

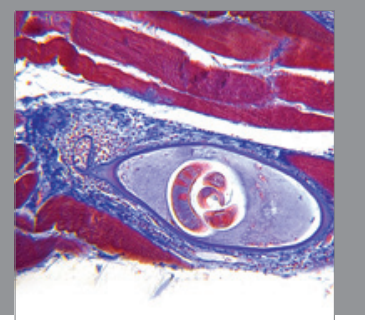

Gastroenterology

Research and Practice
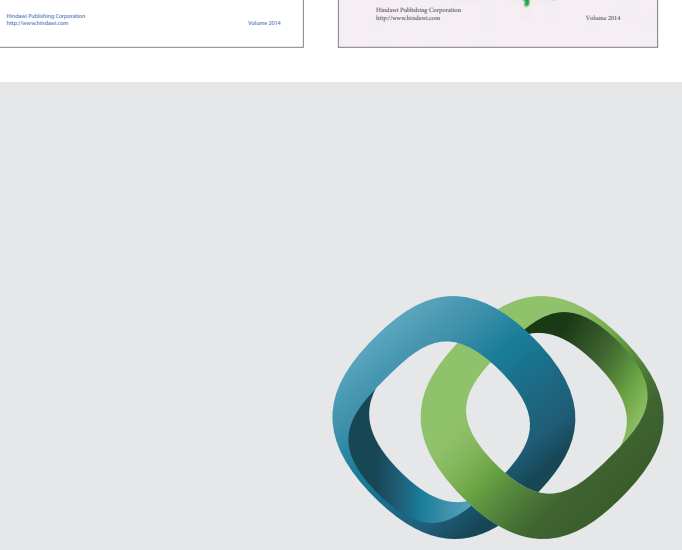

\section{Hindawi}

Submit your manuscripts at

http://www.hindawi.com
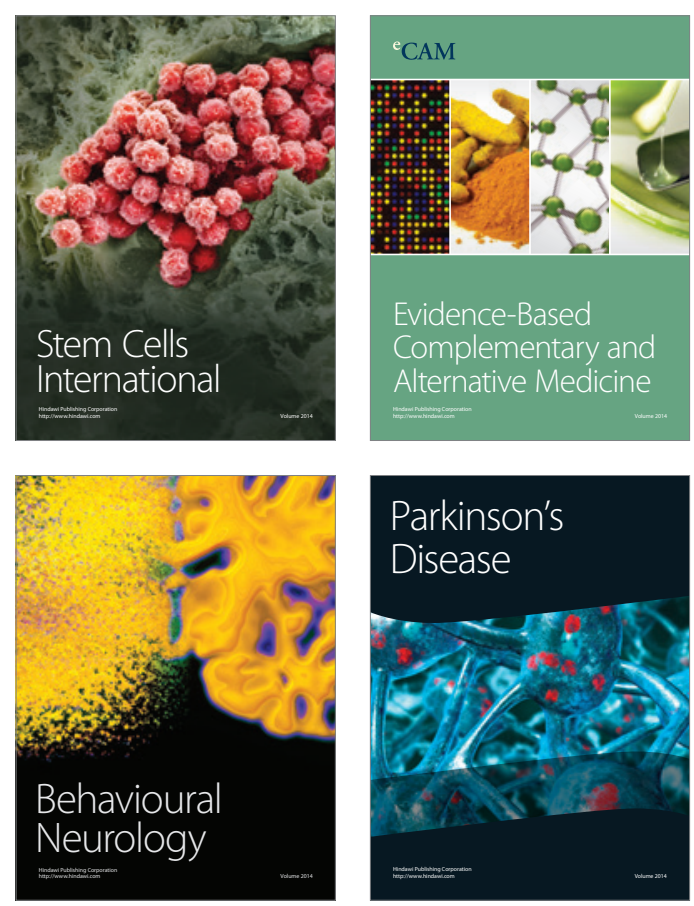

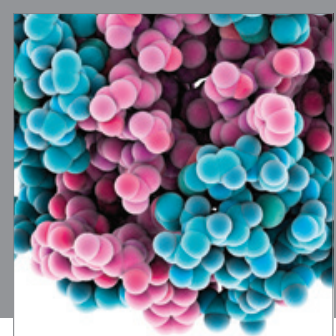

Journal of
Diabetes Research

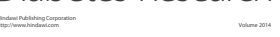

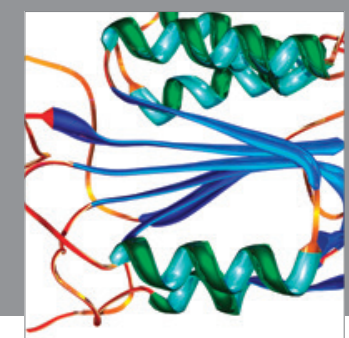

Disease Markers
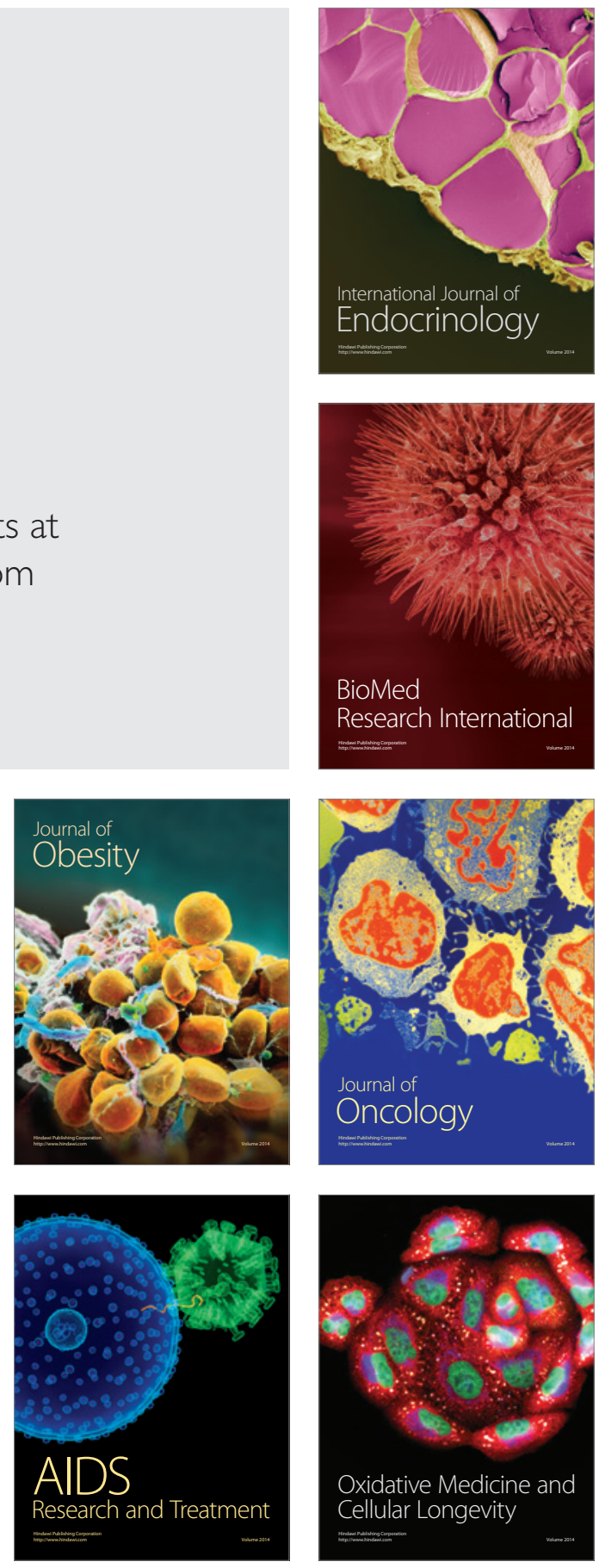